\title{
On the property of "statistical identity" of solutions to some classical problems of the radiative transfer theory
}

\author{
H. V. Pikichyan* \\ Byurakan Astrophysical Observatory, 378433, Aragatsotn District, Armenia
}

\begin{abstract}
A probabilistic interpretation of the classical solution of the diffuse reflection problem (DRP) of radiation from a semi-infinite homogeneous scattering-absorbing medium on the language of random events in the simple case of monochromatic and isotropic scattering is constructed. A certain property of the so-called "statistical identity" is specially defined. By using these two circumstances, it is possible to construct a simple symbolic scheme for the direct transformation of the solution mentioned above in the particular case of DRP into solutions to more general cases of DRP, which taking into account the anisotropy and incoherence of scattering, as well as the temporal dependence of the task on the act of absorption. Moreover, some generalization of the primary scheme makes it possible to directly obtain solutions of the DRP also for nonhomogeneous media and for general case of time dependence (on absorption acts and free flights between them) for the quanta diffusion process. At the same time, both the well-known results of the DRP decisions and some new ones were obtained.
\end{abstract}

Keywords: radiative transfer- probabilistic interpretation-diffuse reflection problem

\section{Introduction and purpose of the work}

In 1942-44 by introducing the principle of invariance and the layer addition method into the theory of radiative transfer, Ambartsumian (Ambartsumian, 1942, 1943a,b, 1944a,b) obtained precise solutions to a number of diffuse reflection problems (DRP) from a semi-infinite medium and reflection-transmission for a layer of finite optical thickness. A significant advantage here in comparison with the traditional approach of applying the integro-differential equation of radiative transfer was to determine the diffuse radiation leaving the medium without the need to find the field inside the medium. Further, these considerations were widely used by many authors to analyze problems of varying complexity. However, it is well known that, both through the radiation transfer equation (Boltzmann's kinetic equation for a photonic gas) and through the application of invariance and layer addition considerations, analytical and numerical-analytical studies of transfer problems are associated with complex mathematical calculations. At the same time, with the complication of physical situations in the studied problems, both the number of independent variables and the cumbersomeness of the mathematical procedures used increase. Therefore, it is very advisable to search for more simplified private methods for analyzing such problems.

The purpose of the presented work is, using the example of a well-studied early DRP from a semi-infinite medium, to illustrate the possibility of obtaining solutions and corresponding functional equations of physically more general and complex DRP by direct transformation of a known solution corresponding to them more particular and simple DRP. At the same time, we will proceed from the probabilistic interpretation and statistical description of transport phenomena using the highlighted below property of the so-called "statistical identity" between the corresponding solutions and functional equations of more "particular" and more "general" problems.

*hovpik@bao.sci.am,hovpik@gmail.com 


\section{Property of statistical identity}

In 1951, Sobolev introduced the concept of the exit probability of an absorbed quantum from the medium and the approach of statistical interpretation of radiative transfer processes (see, for example, (Sobolev, 1956a)). It is well known that this made it very easy to write down some of the relations and integral equations of the radiation transfer theory directly from the probabilistic reasons, bypassing the mathematical calculations of their derivation from the equation of radiative transfer. For example, in the classical DRP of a unit quantum from a semi-infinite scattering-absorbing homogeneous medium, in the case of monochromatic and isotropic scattering, the expression is directly recorded from the probabilistic reasons is

$$
\rho\left(\mu \leftarrow \mu^{\prime}\right)=\int_{0}^{\infty} p(\mu, \tau) \cdot e^{-\frac{\tau}{\mu^{\prime}}} \cdot \frac{d \tau}{\mu^{\prime}} .
$$

Indeed, the probability of diffuse reflection $\rho\left(\mu \leftarrow \mu^{\prime}\right) d \mu$ in the direction of $\mu$, inside the solid angle $2 \pi d \mu$ of a unit quantum falling on the boundary of the medium, from the direction arccos $\mu^{\prime}$ with respect to its normal, is formed from the sequential realization of three independent events. The first event - entering the medium quantum first with probability $e^{-\frac{\tau}{\mu^{\prime}}}$ flies some optical distance $\frac{\tau}{\mu^{\prime}}$ freely. The second event - then the quantum is absorbed in the "infinitely thin" layer $d \tau$ of the depth $\tau$, with a probability of $\frac{d \tau}{\mu^{\prime}}$. As a result, after this first act of absorption, there is a quantum absorbed at depth $\tau$ in the medium, which with probability $p(\mu, \tau) d \mu$ (in the direction of $\mu$, inside the solid angle $2 \pi d \mu$ ) will realize the final third "complex event" - the process of its exit through the outer boundary of the semi-infinite medium after arbitrary wanderings in it. Since the above-mentioned first act of absorption can occur at any current value of $\tau$, the integral over the entire interval of change of these values $[0, \infty]$ is obviously taken. For an unknown in (1) quantity $p(\mu, \tau)$ in the same simple way - directly for probabilistic reasons, the integral equation defining it is written (Sobolev, 1956a).

It is very important to note that the underlying statistical sequence of the three random events listed above is universal, that is, it is valid outside the specific physical properties of the elementary act of scattering. Therefore, the structure of expression (1) will not change if, taking into account the corresponding new physical variables of the problem. This expression is also rewritten for more general assumptions about the properties of the elementary act of scattering. In the case of noncoherent non-isotropic scattering, the expression (1) will take the form

$$
\rho\left(\mu, x \leftarrow \mu^{\prime}, x^{\prime} ; \varphi-\varphi^{\prime}\right)=\int_{0}^{\infty} p\left(\mu, x \leftarrow \mu^{\prime}, x^{\prime} ; \varphi-\varphi^{\prime} ; \tau\right) \cdot e^{-\alpha\left(x^{\prime}\right) \frac{\tau}{\mu^{\prime}}} \alpha\left(x^{\prime}\right) \cdot \frac{d \tau}{\mu^{\prime}},
$$

where the same three events are presented, indicating only the quantities describing the new physical situation: directions - $(\mu, \varphi),\left(\mu^{\prime}, \varphi^{\prime}\right)$ and the dimensionless frequencies $-x, x^{\prime}$ related to the quant entering and leaving the medium, respectively, as well as the absorption coefficient profile - $\alpha\left(x^{\prime}\right)$. Indeed, the expression (2) is not different in structure from (1). Here, the same three random events appear, only taking into account the characteristics of a new, more complex physical situation.

Definition: The structural unity of the probabilistic interpretation (in the sense of identity and sequence of realized random events) of relations describing different relative to the elementary act of scattering (i.e., simpler and more complex) problems, conditionally called the property of the statistical identity of these relations or tasks.

Obviously, the ratios (1) and (2) in the sense of the formulated definition are statistically identical. 


\section{Probabilistic interpretation of the classical solution of the DRP from a semi-infinite medium}

The classical solution of the DRP from a homogeneous semi-infinite medium in the case of monochromatic and isotropic scattering is of the form (Ambartsumian, 1942, 1943b)

$$
\begin{gathered}
\rho\left(\mu, \mu^{\prime}\right)=\frac{\lambda}{2} \mu \frac{\varphi(\mu) \varphi\left(\mu^{\prime}\right)}{\mu+\mu^{\prime}}, \quad \varphi(\mu)=1+\int_{0}^{1} \rho\left(\mu, \mu^{\prime}\right) d \mu^{\prime}, \\
\varphi(\mu)=1+\frac{\lambda}{2} \mu \int_{0}^{1} \frac{\varphi\left(\mu^{\prime}\right)}{\mu+\mu^{\prime}} d \mu^{\prime} .
\end{gathered}
$$

To use in (3), similarly to (1) and (2), the statistical identity property defined above, it is first necessary to find out the probabilistic meaning of the solution itself (3). It's not hard to see if it can be rewritten as

$$
\left(\frac{1}{\mu}+\frac{1}{\mu^{\prime}}\right) \cdot \rho\left(\mu, \mu^{\prime}\right)=p(0, \mu) \cdot \tilde{p}\left(0,-\mu^{\prime}\right)
$$

where the factors

$$
\tilde{p}\left(0,-\mu^{\prime}\right)=\frac{\varphi\left(\mu^{\prime}\right)}{\mu^{\prime}}, \quad p(0, \mu)=\frac{\lambda}{2} \varphi(\mu)
$$

describe the probabilities of two "optically inverse" to each other processes. Indeed, the right factor on the right side of $(5)-\tilde{p}\left(0,-\mu^{\prime}\right) d \tau$, represents the probability of absorption (directly or by arbitrary wandering) of a unit quantum on the "infinitely thin" boundary $d \tau$ layer of the medium, provided that this quantum initially fell from the outside to the boundary of the medium from the direction of $\mu^{\prime}$ to its external normal (Pikichyan 1980). The left multiplier - $p(0, \mu) d \mu$, represents the probability of the quantum initially absorbed at the boundary of the medium, which arbitrarily (after re-emission - directly, or by generally speaking multiple scatterings) will leave the medium in the direction of $\mu$, inside the solid angle $2 \pi d \mu$ ( $\lambda$ - the probability of re-emission of the quantum after absorption). The probabilistic interpretation of formula (5), taking into account the above, states the equivalence of two complex events - processes represented in two parts of this equation. The left part in the form of two multipliers represents the probability of joint realization of two random events. The first is the direct absorption in an infinitely thin boundary layer of the medium of a quantum with a probability of $\left(\frac{1}{\mu}+\frac{1}{\mu^{\prime}}\right) d \tau$ when it enters the medium or when it leaves it. The second is the diffuse reflection of the quantum from the medium, after arbitrary wanderings in it, with a probability of $\rho\left(\mu, \mu^{\prime}\right) d \mu$. The right part of (5) describes the joint sequential implementation of the two mentioned above, generally speaking, diffusion processes with probabilities $\tilde{p}\left(0,-\mu^{\prime}\right) d \tau$ and $p(0, \mu) d \mu$.

\section{Scheme of statistical identity for the DRP}

The above statistical interpretation of the DRP (5) in the language of random events is generally more appropriate to present in the form of a simple symbolic scheme

$$
(a+\tilde{a}) \cdot R=\langle A \cdot \tilde{A}\rangle=Q .
$$

Here, the values $a, \tilde{a}$ describe the probabilities of events: single elementary acts of direct absorption of the quantum at the boundary of the medium, $R$ represents the complete process of diffuse reflection of the quantum from the medium, and the bracket $\langle A \cdot \tilde{A}\rangle$ represents the sequence of two described above - optically inverse to each other, generally speaking, diffusion processes $\tilde{A}$ and $A$. Accounting for changes in physical parameters in the elementary act (more generally than (5)) of scattering, on which the necessary integrations should be carried out, is indicated in parentheses - $\langle\ldots\rangle$ (see below). The right part of (7) can be perceived as a single specific statistical process $Q$ where the quantum in its path necessarily undergoes, generally speaking, an intermediate -"diffusion" act of scattering at 


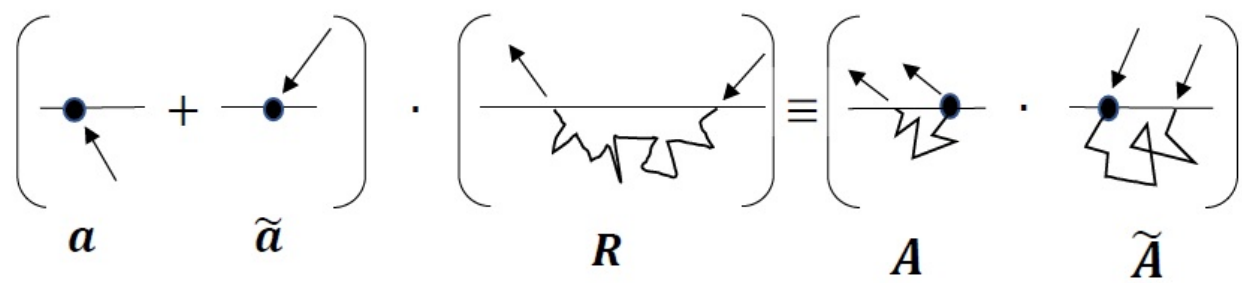

Figure 1.

the boundary of the medium. The formula (7), with the corresponding letter designations of events, in a diagramic way is shown in Fig. 1. The horizontal line indicates the boundary of the medium, below it a semi-infinite scattering-absorbing medium is implied, broken ones symbolically indicate the trajectories of the quantum, the points are the act of absorbing the quantum, and the arrows are the initial or final directions of motion of the quantum.

\section{Examples of the application of the statistical identity property in the DRP}

Here are examples of the application of the statistical identity property according to the scheme (7) with various assumptions more general than (5) regarding the elementary act of scattering. It is important to note that the corresponding symmetry of the diffuse reflection function, deriving, as is well known, from the identical symmetry of the elementary act of scattering, is taken into account in all DRP below. The expression (3) in the case of non-coherent isotropic scattering, with the frequencies redistribution function $r\left(x^{\prime \prime}, x^{\prime}\right)$ of radiation, by means of $(7)$ is directly transformed into a solution of known from (Pikichyan, 1978, 1980)

$$
\begin{aligned}
{\left[\frac{\alpha(x)}{\mu}+\frac{\alpha\left(x_{0}\right)}{\mu_{0}}\right] \rho\left(\mu, x \leftarrow \mu_{0}, x_{0}\right) } & =\frac{\lambda}{2 \mu_{0}} \iint_{-\infty-\infty}^{+\infty+\infty} \varphi\left(\mu, x ; x^{\prime \prime}\right) r\left(x^{\prime \prime}, x^{\prime}\right) \varphi\left(\mu_{0}, x_{0} ; x^{\prime}\right) d x^{\prime \prime} d x^{\prime}, \\
\varphi\left(\mu, x ; x^{\prime \prime}\right) & =\delta\left(x-x^{\prime \prime}\right)+\int_{0}^{1} \rho\left(\mu, x \leftarrow \mu^{\prime \prime}, x^{\prime \prime}\right) d \mu^{\prime \prime},
\end{aligned}
$$

which was obtained earlier by the standard technique of the principle of invariance. In the more general case of redistribution of radiation by frequencies and directions (i.e., in non-coherent nonisotropic scattering), the use of scheme (7) directly leads to the functional equation

$$
\begin{gathered}
\frac{4 \pi \mu^{\prime}}{\lambda}\left[\frac{\alpha(x)}{\mu}+\frac{\alpha\left(x^{\prime}\right)}{\mu^{\prime}}\right] \rho\left(M, M^{\prime}\right)=r\left(M,-M^{\prime}\right)+ \\
\iiint \rho\left(M, M^{\prime \prime \prime}\right) r\left(-M^{\prime \prime \prime} \leftarrow M^{\prime}\right) d^{3} M^{\prime \prime \prime}+\iiint r\left(M, M^{\prime \prime}\right) \rho\left(M^{\prime}, M^{\prime \prime}\right) d^{3} M^{\prime \prime}+ \\
\iiint \iiint\left[\rho\left(M, M^{\prime \prime \prime}\right) r\left(-M^{\prime \prime \prime}, M^{\prime \prime}\right) \rho\left(M^{\prime}, M^{\prime \prime}\right)\right] d^{3} M^{\prime \prime} d^{3} M^{\prime \prime \prime}, \\
\pm M \equiv\{x, \pm \mu, \varphi\}, \iiint d^{3} M \equiv \int_{-\infty}^{+\infty} \ldots d x \int_{0}^{2 \pi} \ldots d \varphi \int_{0}^{1} \ldots d \mu,
\end{gathered}
$$

which was obtained in (Yengibaryan \& Nikoghosyan, 1972) by applying the principle of invariance. This is the "non-coherent analogue" of Ambartsumian's functional equation obtained by him in the case of anisotropic monochromatic scattering in pioneer work (Ambartsumian, 1943a). In a nonstationary DRP of an "instantly emitting external source", taking into account the time losses only for 
acts of absorption-re-emission of the quantum, the scheme (7) directly gives a solution that coincides in form with ours, obtained by the wound in the work (Ueno, 1962):

$$
\begin{gathered}
\left(\frac{1}{\mu}+\frac{1}{\mu_{0}}\right) \rho\left(\mu, \mu_{0} ; u-u_{0}\right)=\frac{\lambda}{2 \mu_{0}} \int_{u_{0}}^{u} e^{-(u-z)} d z \int_{u_{0}}^{z} \varphi\left(\mu ; z-u^{\prime}\right) \varphi\left(\mu_{0} ; u^{\prime}-u_{0}\right) d u^{\prime}, \\
\varphi\left(\mu ; u-u_{0}\right)=\delta\left(u-u_{0}\right)+\frac{\lambda}{2} \mu \int_{u_{0}}^{u} e^{-(u-z)} d z \int_{u_{0}}^{z} \varphi\left(\mu ; z-u^{\prime}\right) d u^{\prime} \int_{0}^{1} \frac{\varphi\left(\mu^{\prime} ; u^{\prime}-u_{0}\right)}{\mu+\mu^{\prime}} d \mu^{\prime} .
\end{gathered}
$$

for another special case, the accounting of time costs only for free flights of the quantum between "instantaneous" acts of scattering. Indeed, the wound was aware (see, for example, (Minin, 1988) ) that the solutions to these two special cases of non-stationary DRP coincide in form. Here, the values of $u_{0}$ and $u$ represent the starting and ending moments of the dimensionless entry and output times of the quantum, respectively.

\section{Generalization of the scheme of statistical identity}

Examples of a homogeneous medium were considered above, while in the problem with a time dependence, the case of the time spent only on the act of absorption-re-emission of the quantum was considered. For taken into account both the non-homogeneity of the medium and of time spent on free flights between scattering acts require a generalization of the scheme (7). Let the boundary layer $[\alpha, \alpha+\tau]$ with the current thickness of $\tau$ be highlight in the original semi-infinite inhomogeneous medium $[\alpha, \infty]$ (here $\alpha$ the truncation parameter of the original inhomogeneous medium). The quantum entering the medium let this layer pass freely - without scattering, and reach its lower boundary $\alpha+\tau$. Then consider the DRP of this quantum from the underlying "current" semi-finite medium $[\alpha+\tau, \infty]$ by means of the above-defined process $Q$. After this specific process of diffuse reflection from this "particular" medium, let the quantum pass the $[\alpha, \alpha+\tau]$ layer again without scattering. As a result, we will have a quantum that has finally left the original medium $[\alpha, \infty]$. If, by integrating all the values of $\tau$, all the "current" semi-infinite partial media are now taken into account, then obviously the solution of the original DRP will be obtained. The generalized scheme of statistical identity in this case will take the form

$$
R=[E Q \tilde{E}] \equiv[E\langle A \cdot \tilde{A}\rangle \tilde{E}]
$$

where the values $\tilde{E}$ and $E$ describe the probabilities of free flight when entering and exiting the original medium, and the square brackets describe the integration operation for all values of the parameter $\tau$. It is not difficult to ascertain that in the more specific DRP discussed in paragraph 5, the scheme (13) naturally turns into - (7).

\section{Examples of application of the generalized scheme (13)}

In the DRP of non-homogeneous medium with isotropic scattering equation (13) directly gives a well-known from the works (Bellman \& Kalaba, 1956, Sobolev, 1956b, Sobolev \& Yanovitsky, 1978) solution

$$
\begin{gathered}
\rho\left(\mu, \mu_{0} ; \alpha\right)=\int_{\alpha}^{\infty} \frac{\lambda\left(\alpha^{\prime}\right)}{2} \varphi\left(\mu, \alpha^{\prime}\right) \varphi\left(\mu_{0}, \alpha^{\prime}\right) e^{-\left(\alpha^{\prime}-\alpha\right)\left(\frac{1}{\mu}+\frac{1}{\mu_{0}}\right) \frac{d \alpha^{\prime}}{\mu_{0}},} \\
\varphi\left(\mu, \alpha^{\prime}\right)=1+\int_{0}^{1} \rho\left(\mu, \mu^{\prime} ; \alpha^{\prime}\right) d \mu^{\prime},
\end{gathered}
$$

Where $\lambda\left(\alpha^{\prime}\right)$ is the probability of re-emission of the quantum after absorption at the depth of $\alpha^{\prime}, \alpha-$ is the parameter of truncation of the non-homogeneous medium. In the case of anisotropic scattering 
in an non-homogeneous medium, the scheme (13) directly gives an analogue of Ambartsumian's functional equation:

$$
\begin{gathered}
\rho\left(M, M_{0} ; \alpha\right)=\frac{1}{4 \pi \mu_{0}} \int_{\alpha}^{\infty} \lambda\left(\alpha^{\prime}\right) e^{-\left(\alpha^{\prime}-\alpha\right)\left(\frac{1}{\mu}+\frac{1}{\mu_{0}}\right)} Q\left(M, M_{0} ; \alpha^{\prime}\right) d \alpha^{\prime}, \\
Q\left(M, M_{0} ; \alpha^{\prime}\right)=\chi\left(M,-M_{0} ; \alpha^{\prime}\right)+ \\
\iint \rho\left(M, M^{\prime} ; \alpha^{\prime}\right) \chi\left(-M^{\prime},-M_{0} ; \alpha^{\prime}\right) d^{2} M^{\prime}+\iint \chi\left(M, M^{\prime} ; \alpha^{\prime}\right) \rho\left(M_{0}, M^{\prime} ; \alpha^{\prime}\right) d^{2} M^{\prime}+ \\
\iiint \int\left[\rho\left(M, M^{\prime \prime} ; \alpha^{\prime}\right) \chi\left(-M^{\prime \prime}, M^{\prime} ; \alpha^{\prime}\right) \rho\left(M_{0}, M^{\prime} ; \alpha^{\prime}\right)\right] d^{2} M^{\prime \prime} d^{2} M^{\prime} .
\end{gathered}
$$

Notations are used:

$$
\pm M \equiv\{ \pm \mu, \varphi\}, \quad \iint d^{2} M \equiv \int_{0}^{2 \pi} \ldots d \varphi \int_{0}^{1} \ldots d \mu, f\left(M, M^{\prime}\right) \equiv f\left(\mu, \mu^{\prime} ; \varphi-\varphi^{\prime}\right) .
$$

In the general problem with time dependence in the mutual accounting of time costs both in the acts of scattering and in free flights between them in the case of monochromatic and isotropic scattering of radiation in the homogeneous medium, the ratio (13) directly gives a solution

$$
\begin{aligned}
& \left(\frac{1}{\mu}+\frac{1}{\mu_{0}}\right) \rho\left(\mu, \mu_{0} ; t\right)=\frac{\lambda}{2 \mu_{0}} \int_{0}^{t} K(t-z) d z \int_{0}^{z} \varphi\left(\mu ; z-z^{\prime}\right) \varphi\left(\mu_{0} ; z^{\prime}\right) d z^{\prime}, \\
& \varphi(\mu ; t)=\delta(t)+\frac{\lambda}{2} \mu \int_{0}^{t} K(t-z) d z \int_{0}^{z} \varphi\left(\mu ; z-z^{\prime}\right) d z^{\prime} \int_{0}^{1} \frac{\varphi\left(\mu^{\prime} ; z^{\prime}\right)}{\mu+\mu^{\prime}} d \mu^{\prime} .
\end{aligned}
$$

Here the quantities are: $t$ - is the total time of the quantum in the medium, $t_{1}$ - is the average time the quantum in the absorbed state in a unit scattering act, $t_{2}$-is the average time of the free run of the quantum between the two scattering acts. The core of the equation is obtained in the form of

$$
K(x) \equiv \frac{e^{-\frac{x}{t_{2}}}-e^{-\frac{x}{t_{1}}}}{t_{2}-t_{1}} .
$$

The expressions (19) - (21) are new and represent a direct generalization of the corresponding formulas of Ueno (1962) obtained by him by applying the principle of invariance of Ambartsumian in the special case of taking into account the time costs of the quantum only during free flight between scatters. It is noteworthy that the expressions (19) - (21) in their simplicity differ favorably from the solution of the same general problem obtained by Minin using the Laplace transform (Minin, 1964), see also (Minin, 1988), in which derivatives of the first and second degrees from some auxiliary function of four variables appeared. The solution of the "instantaneous" illumination problem close to the considered (19) - (21), the DRP when illuminating the medium in the "finite time interval" was obtained earlier in the work of Matsumoto (Matsumoto, 1974). Obviously, in order to move from the solution (19) - (21) of the problem of "instantaneous source" to the case with the source of the "finite time interval" of radiation, it is enough to integrate the formulas (19) - (20) according to the corresponding time interval (see (Matsumoto, 1974)) . In the more general case of anisotropic scattering for the "instantaneous" source discussed above, we directly arrive at the expressions by means of scheme (13):

$$
\begin{gathered}
\left(\frac{1}{\mu}+\frac{1}{\mu_{0}}\right) \rho\left(M, M_{0} ; t\right)=\frac{\lambda}{2 \mu_{0}} \int_{0}^{t} K(t-z) Q\left(M, M_{0}, z\right) d z \\
Q\left(M, M_{0}, z\right)=\delta(z) \chi\left(M,-M_{0}\right)+ \\
+\iint \rho\left(M, M^{\prime \prime} ; z\right) \chi\left(-M^{\prime \prime},-M_{0}\right) d^{2} M^{\prime \prime}+\iint \chi\left(M, M^{\prime}\right) \rho\left(M_{0}, M^{\prime} ; z\right) d^{2} M^{\prime}+
\end{gathered}
$$




$$
\iiint \int \chi\left(-M^{\prime \prime}, M^{\prime}\right) d^{2} M^{\prime \prime} d^{2} M^{\prime} \int_{0}^{z} \rho\left(M, M^{\prime \prime} ; z-z^{\prime \prime}\right) \rho\left(M_{0} \leftarrow M^{\prime} ; z^{\prime \prime}\right) d z^{\prime \prime},
$$

where the symbols (18) are used. The expressions (22) - (23) are new and together represent an analogue of the Ambartsumyan's functional equation (Ambartsumian, 1943b) in the non-stationary problem of diffuse reflection from a homogeneous semi-infinite medium in the case of anisotropic scattering, taking into account the time losses both on scattering acts and on free flights between them.

\section{Conclusion}

In the presented work, a probabilistic interpretation of the classical solution of the problem of diffuse reflection of radiation from a semi-infinite homogeneous scattering-absorbing medium is constructed in the language of random events in the simple case of monochromatic and isotropic scattering. A certain property of the so-called "statistical identity" is specially defined. By using these two circumstances, it is possible to construct a simple scheme for the direct transformation of the solution mentioned above in the particular case of the DRP into solutions to more general cases of DRP, taking into account the anisotropy and incoherence of scattering and the temporal dependence of the act of absorption. Moreover, some generalization of the primary scheme also makes it possible to directly obtain solutions to the DRP of non-homogeneous media and the general case of timedependence (acts of absorption and free flights between them) of the process of diffusion of quanta in it. At the same time, both the well-known results of the DRP decisions and some new ones were obtained.

\section{References}

Ambartsumian V. A., 1942, Astronomicheskii Zhurnal (in Russian), 19, No 5, 30

Ambartsumian V. A., 1943a, Jurnal Teoreticheskoy i Eksperimentalnoy Fiziki (in Russian), 13, No. 9-10, 323

Ambartsumian V. A., 1943b, DAN SSSR, 38, No 8, 257

Ambartsumian V. A., 1944a, DAN SSSR (in Russian), 43, No 3, 106

Ambartsumian V. A., 1944b, Izvestiya. AN ArmSSR, Natural Sci. (in Russian), No. 1-2, 31

Bellman R. E., Kalaba R. E., 1956, Proc. Nat. Acad. Sci. USA, 42, 629

Matsumoto M., 1974, Publ. Astron. Soc. Japan, 26, 241

Minin I. N., 1964, DAN SSSR (in Russian), 154, No. 5, 1059

Minin I. N., 1988, Theory of Radiation Transfer in the Atmospheres of Planets. Nauka, Moscow

Pikichyan O. V., 1978, DAN Arm. SSR, (in Russian), 67, No. 3, 151

Pikichyan O. V., 1980, Soobshcheniya BAO (in Russian), 52, 148

Sobolev V. V., 1956a, Transfer of radiant energy in the atmospheres of stars and planets (in Russian). Gostekhizdat, Moscow

Sobolev V. V., 1956b, DAN USSR (in Russian), 111, No. 5, 1000

Sobolev V. V., Yanovitsky E. G., 1978, in Voprosy fiziki i evolutsii kosmosa. Izd. AN ArmSSR, Yerevan

Ueno S., 1962, Journal of math. analysis and applications, 4, 1

Yengibaryan N. B., Nikoghosyan A. G., 1972, DAN AN Arm SSR (in Russian), 54, No. 2, 91 